\title{
Introduction to Minitrack - Mixed, Augmented and Virtual Reality: Services and Applications
}

\author{
Jani Holopainen \\ University of Jyväskylä \\ jamaholo@jyu.fi \\ Osmo Mattila \\ University of Helsinki \\ osmo.mattila@helsinki.fi
}

\author{
Essi Pöyry \\ University of Helsinki \\ essi.poyry@helsinki.fi \\ Juho Hamari \\ Tampere University \\ juho.hamari@tuni.fi
}

Virtual reality (VR) refers to computer hardware and software that generate realistic images, sounds and other sensations and illustrate an immersive environment to simulate a user's physical presence in a virtual environment [1]. Mixed and augmented reality (MR, AR) refer to combining real and virtual environments, contents, and objects [2]. Due to maturing of VR, AR and MR technologies and the increased need for remote working and studying, improved visualization, communication and guiding, as well as risk reduction, the full potential of these technologies is still ahead. Therefore, the need to understand the services and applications these technologies can provide is greater than ever.

For HICSS 2022, the minitrack received nine submissions out of which four papers were accepted. The accepted papers represent a wide array of perspectives in MR service research as well as fields these technologies contribute to; from healthcare to engineering and online commerce, MR technologybased services can make a notable difference to our societies. Next, the accepted papers are introduced.

A paper called Virtual Reality Technologies in Health Care: A Literature Review of Theoretical Foundations by Michael Knop, Caroline Ressing, Marius Mueller, Sebastian Weber, Henrik Freude, and Bjoern Niehaves analyzes 20 empirical and conceptual papers that have studied the use of VR technologies in healthcare. The authors note that only a few of them combined information systems-based theories or concepts to theories of clinical healthcare or interventions to explain why VR technologies might be effective in rehabilitation, therapy, surgery or elsewhere in healthcare. The paper therefore pinpoints an important avenue for future research while highlighting the need for application-oriented research in this area.
In A Comparison of Gesture and Controllerbased User Interfaces for 3D Design Reviews in Virtual Reality, Taneli Nyyssönen, Seppo Helle, Teijo Lehtonen, and Jouni Smed developed and tested a gesture-based user interface for 3D design review purposes and compared it with a controller-based comparison software. Based on the test, the gesturebased UI was considerably less reliable but significantly more immersive than the controllerbased UI. The findings provide a fruitful basis for the design of new 3D modeling applications for example in ship building and other fields of engineering.

In their paper called Haptic Sensory Perception and Customer Experience in a 360-Virtual Store: Does Time Spent Play a Role?, Yuyu Wang, Heli Hallikainen, Nino Ruusunen, and Tommi Laukkanen examine with a large experimental setup the effects of haptic sensory perception on user experience and satisfaction in a 360-virtual store. The authors argue that 360-virtual reality creates opportunities for online retailers in terms of offering consumers with a more immersive and realistic online shopping environment, particularly if they are not in a hurry and are browsing the store without a specific goal in mind.

Finally, in the minitrack's second paper concentrating on the retail context, How Does Extended Reality Influence Consumer Decision Making? The Empirical Evidence from $A$ Laboratory Experiment, Nannan Xi, Elpida Bampouni, and Juho Hamari present a laboratory experiment which compares four kinds of environments (physical reality, augmented reality, virtual reality, and augmented virtuality) with regards to consumer decision making. According to the results, shopping in a VR environment improved 
decision quality but shopping in the physical reality led to highest decision satisfaction.

Together these studies contribute to an exciting new opportunity within MR technology research combining different kinds of technologies, environments and user interfaces to create a seamless and effective user experience. Learning from different application contexts can generate best practices also elsewhere.

\section{References}

[1] Steuer, J. "Defining virtual reality: Dimensions determining telepresence", Journal of Communication, 42, 1992, pp. 73-93.

[2] Milgram, P., Takemura, H., Utsumi, A., \& Kishino. F. "Augmented reality: A class of displays on the reality-virtuality continuum", Proceedings of Telemanipulator and Telepresence Technologies, 1994, pp. 23512334. 